\title{
Finite element analysis of the tibial bone graft in cementless total knee arthroplasty

Koji Totoribe 1* $^{*}$, Etsuo Chosa', Go Yamako², Hiroaki Hamada ${ }^{1}$, Koki Ouchi², Shutaro Yamashita ${ }^{2}$ and Gang Deng ${ }^{2}$

\begin{abstract}
Background: Achieving stability of the tibial implant is essential following cementless total knee arthroplasty with bone grafting. We investigated the effects of bone grafting on the relative micromotion of the tibial implant and stress between the tibial implant and adjacent bone in the immediate postoperative period.

Methods: Tibial implant models were developed using a nonlinear, three-dimensional, finite element method. On the basis of a preprepared template, several bone graft models of varying sizes and material properties were prepared.

Results: Micromotion was larger in the bone graft models than in the intact model. Maximum micromotion and excessive stress in the area adjacent to the bone graft were observed for the soft and large graft models. With hard bone grafting, increased load transfer and decreased micromotion were observed.

Conclusions: Avoidance of large soft bone grafts and use of hard bone grafting effectively reduced micromotion and undue stress in the adjacent area.
\end{abstract}

Keywords: Total knee arthroplasty, Bone graft, Finite element analysis

\section{Background}

Tibial bone defects in total knee arthroplasty (TKA) often are managed with various types of bone grafts, such as solid bone, morselized bone, and combined grafts [1-4]. For optimal results, patients with bone grafts require longterm postoperative therapy. In particular, during weightbearing gait training, the mechanical condition of the grafted bone and micromotion-preventing fixation of the tibial component influence the degree of micromotion [3]. Whether the patient is able to walk safely immediately postoperatively is an important concern after cementless TKA. The finite element method is being used increasingly for biomechanical analysis of tibial component micromotion. Several studies have analyzed the relative micromotion of implanted prostheses using this method [5-8]. We have previously reported that tibial bone graft combined with cortical bone can be expected to reduce micromotion and stress [8]. However, the simulation was not enough to include the characteristics of implant and bone graft materials in the models or to examine the detailed status of contact and stress distribution while considering

\footnotetext{
* Correspondence: totoribe@med.miyazaki-u.ac.jp

${ }^{1}$ Department of Orthopaedic Surgery, Faculty of Medicine, University of

Miyazaki, 5200 Kihara, Kiyotake, Miyazaki 889-1692, Japan

Full list of author information is available at the end of the article
}

micromotion. To our knowledge, no analysis has been conducted considering the actual bone graft size and material properties. We used the finite element method to develop a new precise model of the proximal tibia and tibial implant and meticulously analyzed the biomechanical effects of various types of bone grafts on relative micromotion and stress distribution at the tibial tray-bone interface in the immediate postoperative period.

\section{Methods}

Intact (I) model

To prepare a three-dimensional finite element model, geometric data for the proximal tibia were obtained from 0.6-mm-wide computed tomography (CT) scans of the right tibia of a 40-year-old woman. Before CT scanning, informed consent was obtained from the subject, and the study was approved by our institutional research ethics committee. In the global coordinate system, the long axis of the tibia was the line connecting the center of the ankle joint and the midpoint of the lateral and medial tibial condyles, which was defined as the $z$ axis [9]. The transverse plane ( $x y$-plane) was perpendicular to the long axis. To simplify the preparation of the models, the fibula was ignored (Fig. 1a). The three- 


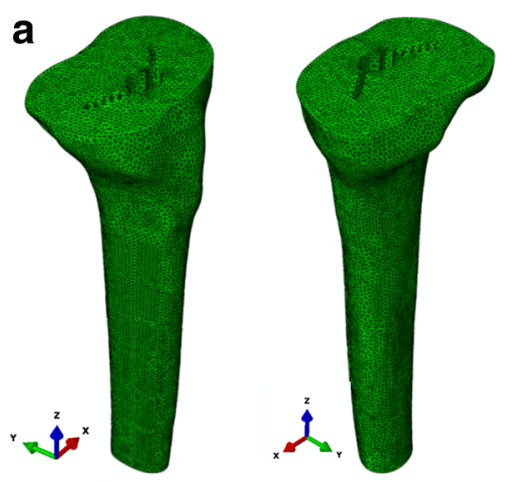

b

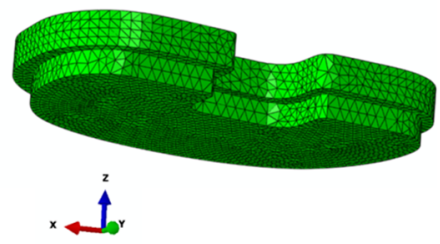

C

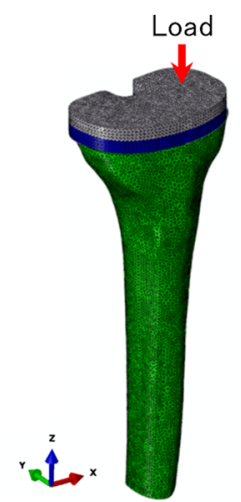

Fig. 1 Detailed finite element model of the proximal tibia and tibial component. The directions of the axes of the global coordinate system are shown. a Intact tibia showing the contact surface with the tibial component. b UHMWPE insert. c Tibial baseplate with keel. $\mathbf{d}$ Load application to the model

dimensional four-noded tetrahedral element was used for modeling cortical and cancellous bone and tibial baseplate. A tibial model comprising a total of 450,697 solid elements was developed (Fig. 1). For development of the models and their subsequent analysis, the multipurpose finite element analysis software ABAQUS/ Standard (Simulia, Tokyo, Japan) was used.

\section{Tibial component}

An appropriate modular tibial baseplate made of cobaltchrome alloy (\#3 size, Osteonics 7000 tibial component; Howmedia Osteonics Corp., Mahwah, NJ, USA), applied in accordance with the manufacturer's guidelines, was considered in this study (Fig. 1c). The geometry of the modular tibial component was obtained by direct measurement. The tibial insert was composed of 8-mm-thick ultra-high molecular weight polyethylene (UHMWPE). The rounded top surface of the UHMWPE was ignored, and a flat surface was defined (Fig. 1b). The inferior surface of the UHMWPE was attached to the tibial baseplate, which was positioned at the geometric center of the tibia, tilted $3^{\circ}$ posteriorly (Fig. $1 \mathrm{~d}$ ).

\section{Bone graft models}

Clinically, bone defect locations in the proximal tibia often are distributed in the posteromedial region. Using the standard tibial model, a bone graft model representative of a posteromedially located bone graft was prepared. The models were graded as large, medium, and small in terms of the bone graft depth $(9,6$, and $3 \mathrm{~mm}$, respectively; Fig. 2). To account for the various techniques used for bone grafting, two types of bone graft models were prepared in the posteromedial portion, with different material properties. The first was a soft bone graft (Fig. 2a-c) in which the defect was filled with a morselized cancellous bone graft, simulating a mechanical worst-case scenario. The second was a hard bone graft, simulating a stronger bone graft, such as tightly impacted morselized cancellous bone grafts than those achieved with simple morselized cancellous bone. Thus, the bone graft models comprised the following six variations: (1) L-S (large bone graft with soft bone), (2) M-S (medium bone graft with soft bone), (3) S-S (small bone graft with soft bone), (4) L-H (large bone graft with hard bone), (5) $\mathrm{M}-\mathrm{H}$ (medium bone graft with hard bone), and (6) S-H (small bone graft with hard bone).

\section{Material properties}

For the tibia, Young's modulus of cortical and cancellous bone was set at 17,000 and $400 \mathrm{MPa}$, with Poisson's ratio of 0.30 [10, 11]. Young's modulus of morselized bone 

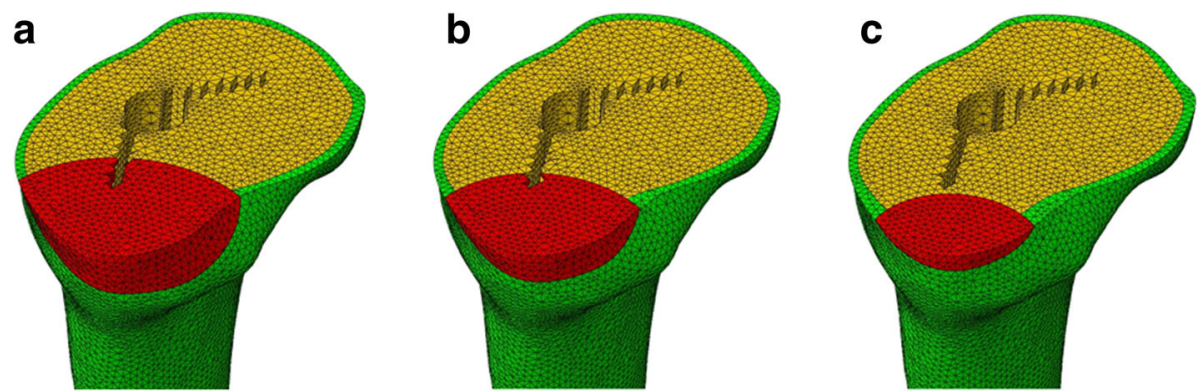

Fig. 2 Finite element models of bone grafts. Posterolateral view of the bone graft area. a Large, $\mathbf{b}$ medium, and $\mathbf{c}$ small bone defects filled with bone graft

graft for bone graft models has been reported as 42-150 $\mathrm{MPa}$ [12-14]. In the present bone graft models, simulating the treatment of bone defects with morselized bone graft, Young's modulus for the bone graft site was set at 42 or $150 \mathrm{MPa}$ with Poisson's ratio of 0.2 , the respective low- and high-strength bone graft values according to the literature.

The cobalt-chrome alloy used for the tibial baseplate component and the UHMWPE used for the tibial insert were given Young's modulus (Poisson's ratio) of 248,000 $\mathrm{MPa}$ (0.30) and $667 \mathrm{MPa}$ (0.46), respectively [11]. The interface between the bone and tibial component was treated as a frictional contact problem, with friction set at 0.2 according to the literature [15].

\section{Boundary and loading conditions}

The inferior surface of the distal tibia was fixed in all directions. The maximum loads used in the analyses were the maximum physiological loads used in previously described experiments [16, 17]. A compressive force of approximately three times the body weight, or $1800 \mathrm{~N}$, was used in this study. Regarding the site of loading, the posteromedial portion was used, as in the previously described experiments $[5,18]$, to simulate a varus alignment. For nonlinear analyses, incremental force control was used in the numerical procedure. Each model was loaded, and the resultant displacement, maximum liftoff, subsidence, and von Mises stress distribution were analyzed and compared.

\section{Results}

For the medial load application shown in Fig. 1d, the stability of the implant and stress distribution are important from the biomechanical viewpoint. Relative micromotion between the tibial tray (Fig. 1c) and tibial bone (Fig. 1a) model at the location of maximum liftoff and at the distal tip of the stem and stress distribution in the tibial bone for the intact and various types of bone graft models shown in Fig. 2 were calculated.

\section{Displacement and micromotion Displacement}

Figure 3 shows the displacement of the tibial tray for all models. Displacement was highest at the periphery of the tibial tray. The medial side of the tibial tray exhibited subsidence at the medial plateau because of the medial load, and the opposite lateral side showed liftoff.

\section{Maximum liftoff}

Figure 4 shows the relative micromotion ( $z$-component) between the tibial tray and tibial bone model at the location of maximum liftoff. The maximum liftoff of the tibial tray in the I model was $14.5 \mu \mathrm{m}$. Compared with the I model, all bone graft models showed increased liftoff: 289,128 , and $47 \%$ in the L-S (largest motion), M-S, and $\mathrm{S}-\mathrm{S}$ (least motion) models, respectively.

The maximum liftoff was smaller in the hard than in the soft bone graft models. Comparing the $\mathrm{L}-\mathrm{S}$ and $\mathrm{L}-\mathrm{H}, \mathrm{M}-\mathrm{S}$ and $\mathrm{M}-\mathrm{H}$, and $\mathrm{S}-\mathrm{S}$ and $\mathrm{S}-\mathrm{H}$ models revealed that the maximum liftoff of the tibial tray was lower in the hard bone graft models by approximately 39,26 , and $12 \%$, respectively, indicating a decrease in the micromotion of hard bone. In the $\mathrm{M}-\mathrm{H}$ and $\mathrm{S}-\mathrm{H}$ models, the magnitudes of liftoff were almost identical and smaller than those in the I model.

\section{Relative micromotion beneath the stem}

Figure 5 shows the relative micromotion between the tibial tray and tibial bone model at the distal stem tip. The tendency toward micromotion beneath the stem was consistent with that toward liftoff. Micromotion beneath the stem in the I model was $68.8 \mu \mathrm{m}$. The increased rate of micromotion beneath the stem was 83 , 41 , and $16 \%$ in $\mathrm{L}-\mathrm{S}$ (largest motion), $\mathrm{M}-\mathrm{S}$, and S-S models (least motion), respectively.

Compared with the soft bone graft models, the magnitudes of micromotion beneath the stem in the hard bone graft models were significantly lower. The $\mathrm{L}-\mathrm{H}$ and $\mathrm{M}-\mathrm{H}$ models showed a larger and the $\mathrm{S}-\mathrm{H}$ 
a

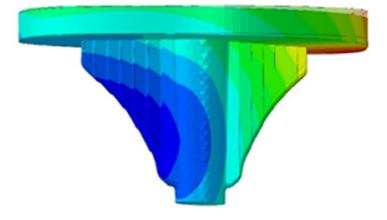

b

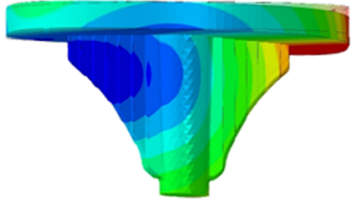

e

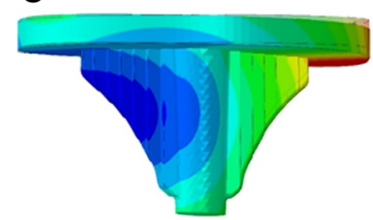

C

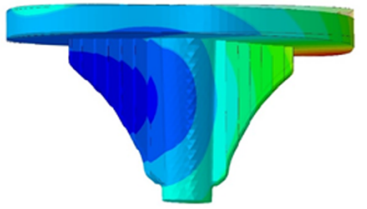

f

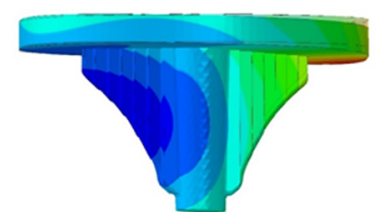

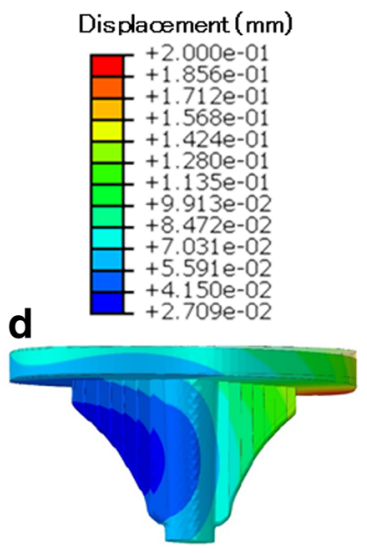

g

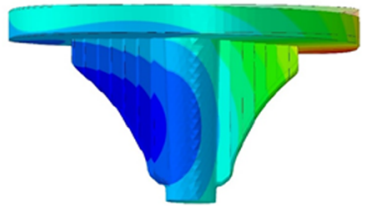

Fig. 3 Resultant displacement of the deformed tibial tray under maximum loading in each model. Contour lines of deformation indicate deformation of the tibial tray. Magnification factor of the displacement $\times 10$. a I (intact). b L-S (large), c M-S (medium), and d S-S (small) bone grafts with soft bone. e $\mathrm{L}-\mathrm{H}$ (large), f M-H (medium), and $\mathbf{g} \mathrm{S}-\mathrm{H}$ (small) bone grafts with hard bone

model a slightly larger magnitude of micromotion than the I model, demonstrating that hard bone grafts have increased stability.

\section{Stress distribution}

Examination of the overall loading of the I model revealed relatively large von Mises stress values in the posteromedial area of the tibial bone, particularly in the cortical bone at the edge of the point of contact. Lateral aspects of the tibia were less loaded than medial regions, except for the lateral keel of the tibial baseplate (Fig. 6a).

In the soft bone graft models, a stress increase was evident in anteromedial and posteromedial cortical bone

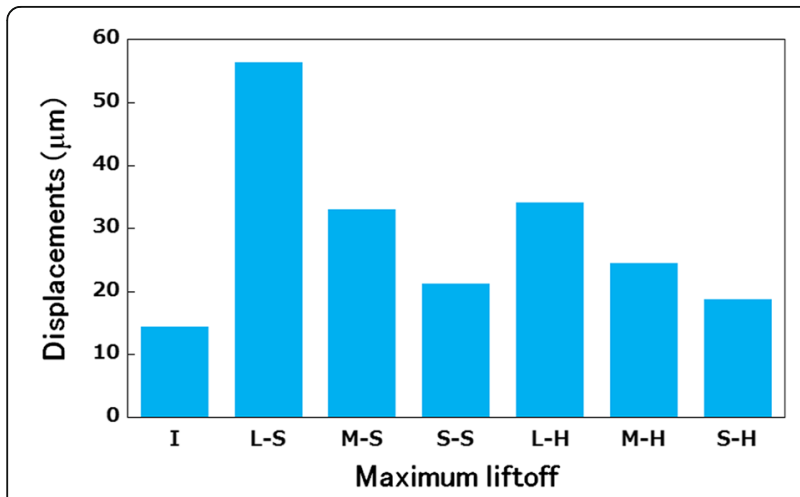

Fig. 4 Maximum liftoff of the tibial tray in each model. I (intact), L-S (large), M-S (medium), and S-S (small) bone grafts with soft bone. L-H (large), $\mathrm{M}-\mathrm{H}$ (medium), and $\mathrm{S}-\mathrm{H}$ (small) bone grafts with hard bone and in the medial host cancellous bone region, including in the keel of the tibial baseplate adjacent to the bone graft, relative to the I model (Fig. 6b-d). In particular, the L-S model exhibited high stress in host cancellous bone. In the hard bone graft models, a stress increase was found in the bone graft area, whereas stresses adjacent to the bone graft area were reduced (Fig. 6e-g).

\section{Discussion}

Medial loading resulted in subsidence of the loaded medial tibial plate and liftoff at the periphery on the opposite, unloaded side. The tilting motion of the tibial components observed in this study implies instability of

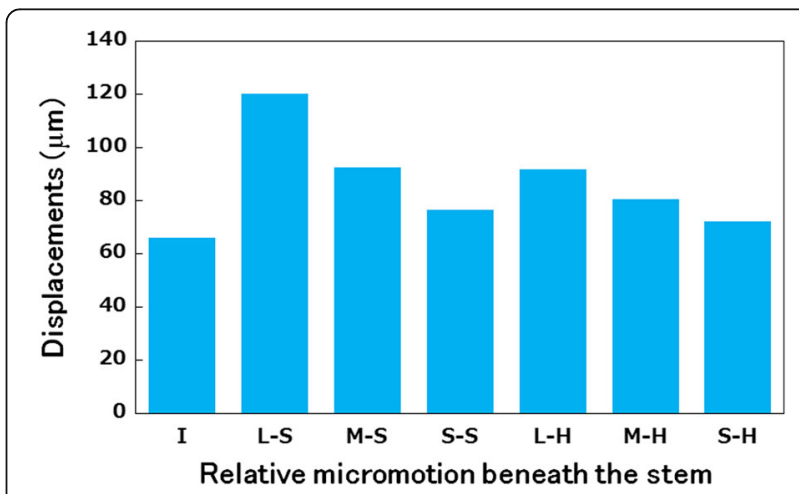

Fig. 5 Relative micromotion beneath the stem in each model. I (intact), L-S (large), M-S (medium), and S-S (small) bone grafts with soft bone. $\mathrm{L}-\mathrm{H}$ (large), $\mathrm{M}-\mathrm{H}$ (medium), and $\mathrm{S}-\mathrm{H}$ (small) bone grafts with hard bone 

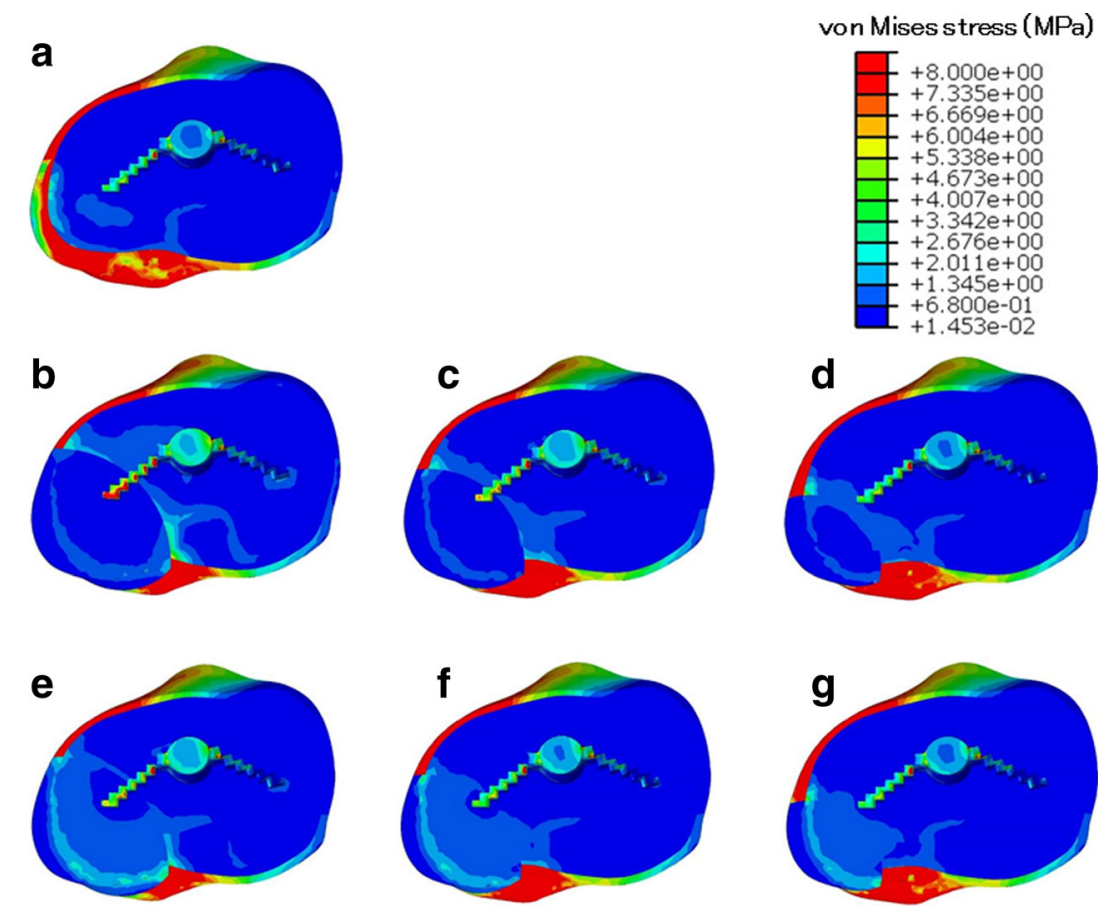

Fig. 6 Top view of von Mises stress distribution on the contact surface of the tibia under maximum loading. a I (intact). $\mathbf{b} L-S$ (large bone graft with soft bone). c M-S (medium bone graft with soft bone). d S-S (small bone graft with soft bone). e L-H (large bone graft with hard bone). $\mathbf{f}$ $\mathrm{M}-\mathrm{H}$ (medium bone graft with hard bone). $\mathbf{g} \mathrm{S}-\mathrm{H}$ (small bone graft with hard bone)

the initial fixation, which could possibly compromise bony ingrowth. The success of cementless implants depends on the ingrowth of bone into the porous coating, which is inhibited by relative micromotion at the implant-bone interface during patient activity $[19,20]$. Excess implant micromotion prevents bone formation within porous-surfaced implants. According to Jasty et al. [21], $150 \mu \mathrm{m}$ of motion results in unstable ingrowth of bone into porous-surfaced implants. Although the $150-\mu \mathrm{m}$ limit often is quoted in the literature, a range of $\pm 50 \mu \mathrm{m}$ was used to assess the sensitivity of the micromotion limit [22]. Our findings showed that soft bone grafting leads to considerable implant micromotion. The relative micromotion observed in the $\mathrm{L}-\mathrm{S}$ model exceeded $100 \mu \mathrm{m}$. This degree of micromotion, if occurring in a clinical situation, may inhibit bony ingrowth and lead to implant loosening. Conversely, use of hard bone decreases micromotion. In all hard bone graft models, the relative micromotion was less than $100 \mu \mathrm{m}$. These results suggested that hard bone grafting is necessary in patients requiring a large bone graft.

Loads were transferred via contact between the tibial baseplate and tibial bone. This contact resulted in an increase in the loading of the medial plateau of the tibia. Analysis of the soft bone graft models revealed considerable unloading of the bone graft area concentrated around the bone graft compared with the I model. Tibial host cancellous bone stresses were reduced by hard bone grafting. The L-S model caused high stress in host cancellous bone. High stress levels, particularly acting on weak cancellous bone, have been implicated as the predominant cause of tibial component loosening [23, 24], and the abnormal stress pattern may cause stress shielding in long-term follow-up [25, 26]. The stress pattern produced by load sharing between the implant and bone suggests that large soft bone grafting should be avoided in favor of grafting with hard bone to eliminate excessive stress in host cancellous bone.

In the previous report, the maximum liftoff and relative micromotion were approximately $2-10$ times higher than those in this study and suggested that bone grafting causes excessive instability [8]. However, from a modeling viewpoint, the shape of the keel of the tibial baseplate was simple, and the roughness of the keel surface to improve fixation was ignored, treating the interface between the bone and implant as a frictionless contact in the previous report. Furthermore, the graft size and material properties were impractical. However, in this study, the shape of the keel designs was precise, treating the interface between the implant and bone as a frictional contact to provide additional stability, in addition to considering realistic graft size and material properties. 
We believe that these distinct differences are the main reasons that affected the results.

With regard to the elastic modulus of bone graft materials, several studies have demonstrated that material stiffness and strength vary substantially according to age, diagnosis, and composition of cancellous and corticocancellous bone and that improved fixation can be achieved by impaction during bone grafting [13, 14, 27-29]. In this study, hard bone models showed lower relative micromotion than soft bone grafting models, suggesting that increased stiffness of the bone graft material by impaction led to decreased micromotion and improved the initial fixation of the tibial baseplate. In clinical cases where the quality of host bone varies substantially between patients, the appropriate bone, including impacted bone, should be grafted into bone defects to achieve optimal stability and a good clinical outcome [4, 30, 31].

With respect to eccentric loading, the finite element analysis of bone grafts undertaken in this study represented the worst-case scenario. Our results suggested that grafting defects with hard bone is more likely to yield better fixation especially for larger, posteromedial defects. During surgery, mechanically effective fixation can be achieved by impaction according to the bone graft material used and type of tibial defects. However, this study did not consider changes in mechanical conditions because of bone remodeling, ingrowth, or stress relaxation. Thus, the qualitative trends outlined here offer an approximation of what occurs during the immediate postoperative phase. This study was designed to provide a foundation for a biomechanical rationale that will support selection of treatment. Because the severity of tibial bone defects and range of bone grafts vary in the clinical setting, care should be taken when interpreting these results. Considering these limitations, combining our results with those of previous cadaveric studies may enhance the clinical use of this technique.

\section{Conclusions}

The purpose of this study was to investigate the effects of bone grafting on the relative micromotion of the tibial implant and stress between the tibial implant and adjacent bone. Maximum micromotion and excessive stress in the host bone area adjacent to the bone graft were observed for the soft and large graft models. On the basis of these biomechanical findings, avoidance of large soft bone grafts and use of hard bone grafting will produce better clinical outcomes.

\section{Abbreviations}

CT: Computed tomography; I: Intact; $L-H$ : Large bone graft with hard bone; L-S: Large bone graft with soft bone; M-H: Medium bone graft with hard bone; M-S: Medium bone graft with soft bone; S-H: Small bone graft with hard bone; S-S: Small bone graft with soft bone; TKA: Total knee arthroplasty; UHMWPE: Ultra-high molecular weight polyethylene

\section{Acknowledgements}

We would like to thank Enago (www.enago.jp) for the English language review.

\section{Funding}

This work was supported in part by a grant from the Ministry of Education, Culture, Sports, Science and Technology of Japan.

\section{Availability of data and materials}

Please contact the author for data requests.

\section{Authors' contributions}

$\mathrm{KT}$, EC, and $\mathrm{HH}$ contributed to the conception and design and data analysis and interpretation. GY, KO, SY, and GD designed the computer model and performed the finite element analysis. All authors helped to draft the manuscript. All authors read and approved the final manuscript.

Ethics approval and consent to participate

This study was approved by the Ethics Committee of the University of Miyazaki.

Informed consent to participate in the study was obtained from the participant.

\section{Competing interests}

The authors declare that they have no competing interests.

\section{Publisher's Note}

Springer Nature remains neutral with regard to jurisdictional claims in published maps and institutional affiliations.

\section{Author details}

'Department of Orthopaedic Surgery, Faculty of Medicine, University of Miyazaki, 5200 Kihara, Kiyotake, Miyazaki 889-1692, Japan. ²Department of Mechanical Design Systems, Faculty of Engineering, University of Miyazaki, 1-1 Gakuen Kibana-dai-nishi, Miyazaki 889-2192, Japan.

Received: 21 February 2018 Accepted: 7 May 2018

Published online: 16 May 2018

\section{References}

1. Dorr LD, Ranawat CS, Sculco TA, McKaskill B, Orisek BS. Bone graft for tibial defects in total knee arthroplasty. Clin Orthop Relat Res. 1986;205:153-65.

2. Görlich Y, Lebek S, Reichel H, Görlich Y, Lebek S, Reichel H. Substitution of tibial bony defects with allogeneic and autogeneic cancellous bone: encouraging preliminary results in 18 knee replacements. Arch Orthop Trauma Surg. 1999:119:220-2

3. Bloebaum RD, Koller KE, Willie BM, Hofmann AA. Does using autograft bone chips achieve consistent bone ingrowth in primary TKA? Clin Orthop Relat Res. 2012;470:1869-78.

4. Naim S, Toms AD. Impaction bone grafting for tibial defects in knee replacement surgery results at two years. Acta Orthop Belg. 2013;79:205-10.

5. Hashemi A, Shirazi-Adl A. Finite element analysis of tibial implants-effect of fixation design and friction model. Comput Methods Biomech Biomed Engin. 2000;3:183-201.

6. Shirazi-Adl A, Patenaude O, Dammak M, Zukor D. Experimental and finite element comparison of various fixation designs in combined loads. J Biomech Eng. 2001;123:391-5.

7. Fitzpatrick CK, Hemelaar P, Taylor M. Computationally efficient prediction of bone-implant interface micromotion of a cementless tibial tray during gait. J Biomech. 2014;47:1718-26.

8. Totoribe K, Chosa E, Sonoda N, Watanabe S, Goto K, Tajima N. Effect of tibial bone graft on the stability in total knee arthroplasty. JJCBM (in Japanese). 2003:24:299-303.

9. Tiftikçi U, Serbest S, Burulday V. Can Achilles tendon be used as a new distal landmark for coronal tibial component alignment in total knee replacement surgery? An observational MRI study. Ther Clin Risk Manag. 2017;13:81-6.

10. Chan Â, Gamelas J, Folgado J, Fernandes PR. Biomechanical analysis of the tibial tray design in TKA: comparison between modular and offset tibial trays. Knee Surg Sports Traumatol Arthrosc. 2014;22:590-8.

11. Brihault J, Navacchia A, Pianigiani S, Labey L, De Corte R, Pascale V, Innocenti B. All-polyethylene tibial components generate higher stress and 
micromotions than metal-backed tibial components in total knee arthroplasty. Knee Surg Sports Traumatol Arthrosc. 2016;24:2550-9.

12. Voor MJ, White JE, Grieshaber JE, Malkani AL, Ullrich CR. Impacted morselized cancellous bone: mechanical effects of defatting and augmentation with fine hydroxyapatite particles. J Biomech. 2004;37:1233-9.

13. Brodt MD, Swan CC, Brown TD. Mechanical behavior of human morselized cancellous bone in triaxial compression testing. J Orthop Res. 1998;16:43-9.

14. Voor MJ, Nawab A, Malkani AL, Ullrich CR. Mechanical properties of compacted morselized cancellous bone graft using one-dimensional consolidation testing. J Biomech. 2000;33:1683-8.

15. Kelly N, Cawley DT, Shannon FJ, McGarry JP. An investigation of the inelastic behaviour of trabecular bone during the press-fit implantation of a tibia component in total knee arthroplasty. Med Eng Phys. 2013;35:1599-606.

16. Collins JJ. The redundant nature of locomotor optimization laws. J Biomech. 1995;28:251-67.

17. Taylor SJ, Walker PS, Perry JS, Cannon SR, Woledge R. The forces in the distal femur and the knee during walking and other activities measured by telemetry. J Arthroplast. 1998;13:428-37.

18. Ahir SP, Blunn GW, Haider $\mathrm{H}$, Walker PS. Evaluation of a testing method for the fatigue performance of total knee tibial trays. J Biomech. 1999; 32:1049-57.

19. Haddad RJ Jr, Cook SD, Thomas KA. Biological fixation of porous-coated implants. J Bone Joint Surg Am. 1987;69:1459-66.

20. Mavrogenis AF, Dimitriou R, Parvizi J, Babis GC. Biology of implant osseointegration. J Musculoskelet Neuronal Interact. 2009;9:61-71.

21. Jasty M, Bragdon C, Burke D, O'Connor D, Lowenstein J, Harris WH. In vivo skeletal responses to porous-surfaced implants subjected to small induced motions. J Bone Joint Surg Am. 1997;79:707-14.

22. Zimmerman WF, Miller MA, Cleary RJ, Izant TH, Mann KA. Damage in total knee replacements from mechanical overload. J Biomech. 2016;49:2068-75.

23. Bourne RB, Finlay JB. The influence of tibial component intramedullary stems and implant-cortex contact on the strain distribution of the proximal tibia following total knee arthroplasty. Clin Orthop Relat Res. 1986;208:95-9.

24. Durig N, Pace T, Broome B, Osuji O, Harman MK. Clinical outcomes of tibial components with modular stems used in primary TKA. Adv Orthop. 2014; 2014:651279.

25. Hayakawa K, Date H, Tsujimura S, Nojiri S, Yamada H, Nakagawa K. Mid-term results of total knee arthroplasty with a porous tantalum monoblock tibial component. Knee. 2014:21:199-203.

26. Jaroma A, Soininvaara T, Kröger H. Periprosthetic tibial bone mineral density changes after total knee arthroplasty. Acta Orthop. 2016;87:268-73.

27. Toms AD, McClelland D, Chua L, de Waal Malefijt M, Verdonschot N, Jones $\mathrm{RS}$, Kuiper J-H. Mechanical testing of impaction bone grafting in the tibia: initial stability and design of the stem. J Bone Joint Surg Br. 2005;87:656-63.

28. Ullmark G, Nilsson O. Impacted corticocancellous allografts: recoil and strength. J Arthroplast. 1999;14:1019-23.

29. Verdonschot $N$, van Hal CT, Schreurs BW, Buma P, Huiskes R, Slooff TJ Time-dependent mechanical properties of HA/TCP particles in relation to morsellized bone grafts for use in impaction grafting. J Biomed Mater Res. 2001:58:599-604.

30. Kharbanda Y, Sharma M. Autograft reconstructions for bone defects in primary total knee replacement in severe varus knees. Indian J Orthop. 2014;48:313-8

31. Toms AD, Barker RL, McClelland D, Chua L, Spencer-Jones R, Kuiper JH. Repair of defects and containment in revision total knee replacement: a comparative biomechanical analysis. J Bone Joint Surg Br. 2009;91:271-7.

\section{Ready to submit your research? Choose BMC and benefit from:}

- fast, convenient online submission

- thorough peer review by experienced researchers in your field

- rapid publication on acceptance

- support for research data, including large and complex data types

- gold Open Access which fosters wider collaboration and increased citations

- maximum visibility for your research: over $100 \mathrm{M}$ website views per year

At BMC, research is always in progress.

Learn more biomedcentral.com/submissions 